\title{
Testing parity-violating mechanisms with cosmic microwave background experiments
}

\author{
Vera Gluscevic and Marc Kamionkowski \\ California Institute of Technology, Mail Code 350-17, Pasadena, California 91125, USA \\ (Received 5 February 2010; published 24 June 2010)
}

\begin{abstract}
Chiral gravity and cosmological birefringence both provide physical mechanisms to produce parityviolating $T B$ and $E B$ correlations in the cosmic microwave background (CMB) temperature/polarization. Here, we study how well these two mechanisms can be distinguished if nonzero $T B / E B$ correlations are found. To do so, we evaluate the correlation matrix, including new $T B-E B$ covariances. We find that the effects of these two mechanisms on the CMB are highly orthogonal, and can thus be distinguished fairly well in the case of a high-signal-to-noise detection of $T B / E B$ correlations. Appendix $\mathrm{B}$ evaluates the relative sensitivities of the $B B, T B$, and $E B$ signals for detecting a chiral gravitational-wave background.
\end{abstract}

DOI: $10.1103 /$ PhysRevD.81.123529

PACS numbers: $98.80 .-\mathrm{k}, 98.70 . \mathrm{Vc}$

\section{INTRODUCTION}

Both inflation [1] and late-time cosmic acceleration [2] require new physics beyond general relativity and the standard model (SM) of particle physics. Since the SM violates parity $(P)$ within the weak sector and is presumably only a low-energy limit of a grand unified theory, it is natural to inquire whether there are manifestations of $P$ violation in the new physics responsible for cosmic inflation and/or late-time acceleration.

For example, a coupling of the quintessence field to the pseudoscalar of electromagnetism would manifest itself as cosmological birefringence (CB) [3], a rotation of the linear polarization of electromagnetic waves as they propagate through the Universe. Parity violation has been introduced in inflation through modifications of gravity that produce a difference in the amplitude of right $(R)$ and left $(L)$ circularly polarized gravitational waves $(\mathrm{GWs})$ in the inflationary GW background. These include the addition of Chern-Simons terms to the Einstein-Hilbert action [4]; chiral gravity, wherein there is a different Newton's constant for $R$ and $L$ gravitational waves [5]; and gravity at a Lifshitz point [6]. We refer collectively to these inflationary mechanisms as chiral gravity.

Since the CMB polarization can be decomposed into two modes of opposite parity $-E$ modes, or the gradient part, and $B$ modes, or the curl part $[7,8]-a$ crosscorrelation between the $E$ and $B$ modes would, if detected, be a sign of parity violation [4]; and similarly for a correlation between the temperature $(T)$ and the $B$ mode. Chiral GWs induce $T B / E B$ correlations at the CMB last scattering surface (LSS) $[4,5]$, while CB induces $P$ violation by rotating the primordial polarization afterwards $[4,9]$.

An early analysis of CMB data suggested a possible CB with rotation angle $\sim 6^{\circ}[10]$, but current constraints are less than a few degrees [11,12]. Reference [13] showed that WMAP does not have enough sensitivity to test chiral gravity and discussed prospects for detection of chiral GWs with Planck and CMBPol.

In this paper, we quantify how well the effects of $\mathrm{CB}$ and chiral gravity can be distinguished, in case of a positive detection of $E B / T B$ correlations. We find that the effects of these two mechanisms are orthogonal to a very high degree, and we show that the earlier tentative detections of $\mathrm{CB}$, if true, could not have been attributed to chiral gravity. We perform these forecasts for WMAP [14], SPIDER [15], Planck [16], CMBPol (EPIC) [17], and a cosmic-variancelimited experiment.

The plan of this paper is as follows: In Sec. II, we forecast the sensitivity of $\mathrm{CMB}$ experiments to gravitational chirality and in Sec. III to CB. Section IV calculates how well the two effects can be distinguished, and in Sec. V we make concluding remarks. In Appendix A, we derive the elements of the power-spectra covariance matrix, and in Appendix B, we evaluate the relative sensitivities of the $B B, T B$, and $E B$ signals to a chiral gravitational-wave background, finding that the best sensitivity comes from the $B B$ signal, in disagreement with an earlier claim [5].

\section{CONSTRAINING GRAVITATIONAL CHIRALITY}

\section{A. Effects of gravitational chirality on the CMB polarization}

If linearized gravity prefers one handedness (i.e., if it is chiral), then the power spectra of the $L$ and $R$ GWs may have different amplitudes and thus induce nonvanishing $T B$ and $E B$ correlations at the LSS [4-6]. Measurements of these correlations can provide an estimate of the chiral asymmetry with a variance due to the finite precision of the instrument and cosmic variance $(\mathrm{CV})$.

We first want to quantify the chirality by introducing an appropriate chirality parameter and show how the CMB polarization map depends on this parameter. To have $B$ modes at the LSS, we need primordial GWs, or in other words, a nonzero tensor-to-scalar ratio,

$$
r \equiv A_{t} / A_{s}, \quad A_{t}=\frac{r}{1+r},
$$

where $A_{t}$ and $A_{s}$ are, respectively, the fractional contributions of tensor and scalar modes to the $T T$ quadrupole. 
Each one of the six CMB temperature/polarization power spectra- $T T, E E, B B, T E, T B$, and $E B$ - have a tensor component proportional to $A_{t}$, while $T T, E E$, and $T E$ additionally have a scalar component proportional to $A_{s}$. The tensor-to-scalar ratio $r$ is currently constrained to be $\leq 0.22$ at a $95 \%$ confidence level [12].

The $T B$ and $E B$ power spectra are proportional to the difference of the $L$ - and $R$-mode contributions to the $\mathrm{GW}$ (tensor) power spectra, $P^{t, L}(k)$ and $P^{t, R}(k)$. These $P$-violating power spectra are [13]

$$
C_{l}^{X X^{\prime}}=(4 \pi)^{2} \int k^{2} d k\left[P^{t, L}(k)-P^{t, R}(k)\right] \Delta_{l}^{X}(k) \Delta_{l}^{X^{\prime}}(k),
$$

while the tensor component of a $P$-conserving correlation is

$$
C_{l}^{X X^{\prime}}=(4 \pi)^{2} \int k^{2} d k\left[P^{t, L}(k)+P^{t, R}(k)\right] \Delta_{l}^{X}(k) \Delta_{l}^{X^{\prime}}(k),
$$

where $P^{t, L}(k)$ and $P^{t, R}(k)$ are the $L$ - and $R$-mode power spectra, $\Delta_{l}^{X}(k)$ is the radiation transfer function for $X$, and $X, X^{\prime}=\{T, E, B\}$. Following Ref. [13], we define a chirality parameter $\Delta \chi$ as

$P^{t, L}(k) \equiv \frac{1}{2}(1+\Delta \chi) P^{t}(k), \quad P^{t, R}(k) \equiv \frac{1}{2}(1-\Delta \chi) P^{t}(k)$,

where

$$
P^{t}(k) \equiv P^{t, L}(k)+P^{t, R}(k) .
$$

Maximal $P$ violation occurs when there are GWs of only one handedness; $\Delta \chi=1$ corresponds to fully left-handed, and $\Delta \chi=-1$ to fully right-handed GWs. To illustrate, we show $B B, T B$, and $E B$ power spectra for $r=0.22$ and $\Delta \chi=0.2$ in Fig. 1 .

To calculate the uncertainty with which $\Delta \chi$ can be estimated with different experiments we use a Fishermatrix analysis [18], employing the null hypothesis, $C_{l}^{E B}=C_{l}^{T B}=0$. This ensures that the $T B$ and $E B$ power spectra do not have cross correlations with the other four power spectra. The reciprocal value of the variance $\sigma_{\Delta \chi}^{2}$ is then given by [7]

$$
\sigma_{\Delta \chi}^{-2}=\sum_{l} \sum_{A, A^{\prime}} \frac{\partial C_{l}^{A}}{\partial \Delta \chi} \frac{\partial C_{l}^{A^{\prime}}}{\partial \Delta \chi}\left[\Xi_{l}^{-1}\right]_{A A^{\prime}},
$$

where $A, A^{\prime}=\{T B, E B\}$, and $\Xi_{l}$ is the $T B-E B$ part of the power-spectrum covariance matrix. ${ }^{1}$ This covariance matrix is derived in Appendix A. The partial derivatives in Eq. (6) can be evaluated by noting from Eqs. (2) and (4) that

\footnotetext{
${ }^{1}$ Under the null hypothesis $\Delta \chi=0$, the $2 \times 2 T B-E B$ part of the inverted $6 \times 6$ covariance matrix is the same as the inverse of the $2 \times 2 T B-E B$ matrix.
}

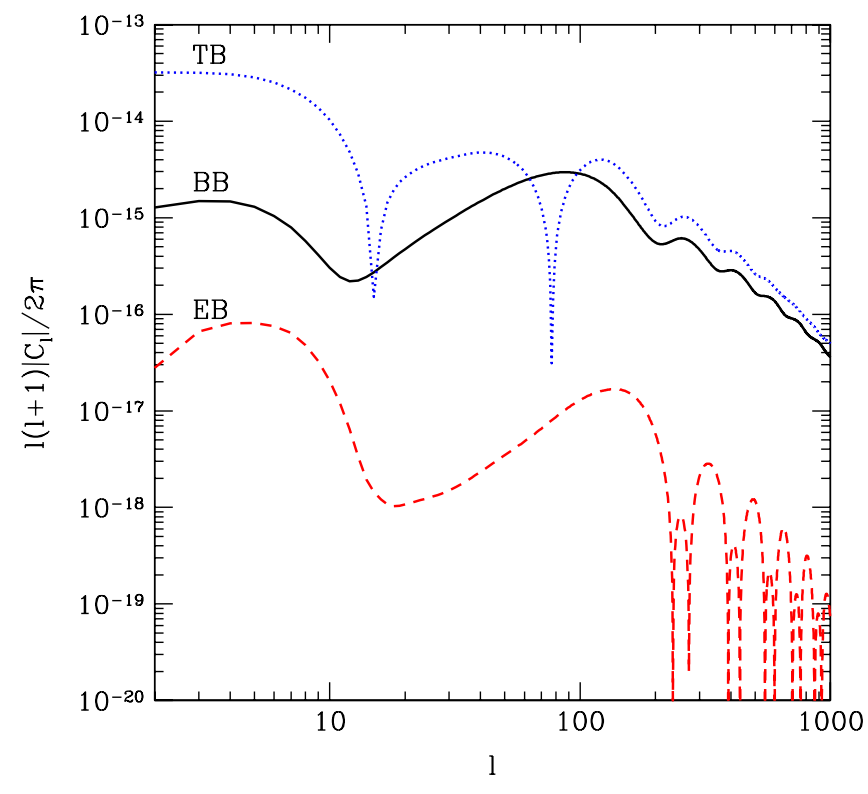

FIG. 1 (color online). $\quad B$-mode power spectra for $r=0.22$ and $\Delta \chi=0.2$.

$$
\left(\partial C_{l}^{T B / E B} / \partial \Delta \chi\right)=C_{l}^{T B / E B}(\Delta \chi=1) .
$$

We obtain the $T B / E B$ power spectra by modifying CMBFAST [19] and using a $\Lambda$ CDM model consistent with WMAP-5 [12] parameters.

\section{B. Numerical results: Forecasts for errors to $\Delta \chi$}

We now forecast the sensitivities to chiral GWs of the following five experiments: (i) WMAP-5, (ii) SPIDER's $150 \mathrm{GHz}$ channel, (iii) Planck's $143 \mathrm{GHz}$ channel, (iv) CMBPol's (EPIC-2m) $150 \mathrm{GHz}$ channel, and (v) a CV-limited experiment. The corresponding instrumental parameters are given in Table I. Note that the noiseequivalent temperature NET is related to the temperature/ polarization pixel-noise variances, $\sigma_{T / P}$, as $\sigma_{T}^{2} / N_{\text {pix }}=$ $(\mathrm{NET})^{2} / t_{\mathrm{obs}}$, where $\sigma_{P}=\sqrt{2} \sigma_{T}$. We take $f_{\text {sky }}^{0}=1.0$ (the fraction of the sky surveyed), and $f_{\text {sky }}=0.7$ (the fraction of the sky used in the analysis), for all experiments, except for SPIDER, where $f_{\text {sky }}^{0}=f_{\text {sky }}=0.5$.

TABLE I. Instrumental parameters from Refs. [15,20-22] for the five experiments considered in this paper. The parameters are the beamwidth $\theta_{\mathrm{FWHM}}$, noise-equivalent temperature NET, and observation time $t_{\mathrm{obs}}$.

\begin{tabular}{lccl}
\hline \hline Instrument & $\theta_{\text {FWHM }}(\operatorname{arc} \min )$ & $\mathrm{NET}(\mu \mathrm{K} \sqrt{\mathrm{s}})$ & $t_{\text {obs }}(\mathrm{yr})$ \\
\hline WMAP-5 & 21 & 650 & 5 \\
SPIDER & 60 & 3.1 & 0.016 \\
Planck & 7.1 & 62 & 1.2 \\
CMBPol & 5 & 2.8 & 4 \\
CV-limited & 5 & 0 & 1.2 \\
\hline \hline
\end{tabular}




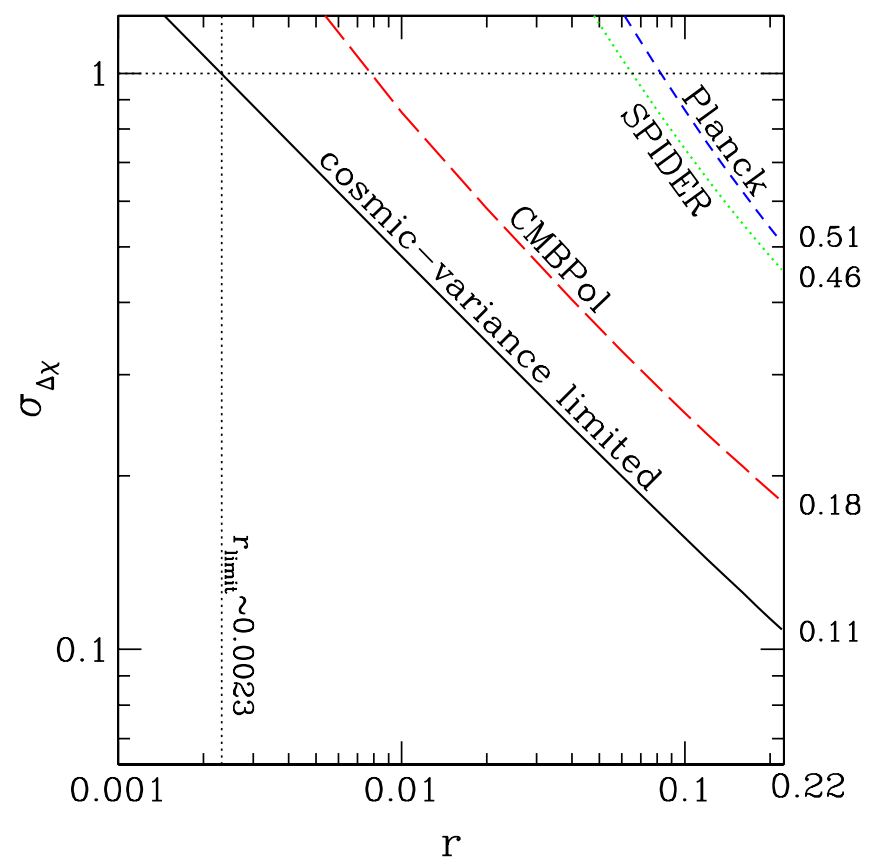

FIG. 2 (color online). $1 \sigma$ error on the gravitational chirality parameter $\Delta \chi$, for four different $\mathrm{CMB}$ experiments, for the fiducial value of $\Delta \chi=0$. The horizontal dotted line is at $\sigma_{\Delta \chi}=$ 1 and represents maximal $P$ violation. In the region above this line, the chirality is nondetectable. The WMAP-5 curve lies entirely above the nondetection line.

Figure 2 shows the $1 \sigma$ error of the estimate of $\Delta \chi$ as a function of tensor-to-scalar ratio $r$. The error increases with decreasing $r$, which implies the existence of a critical value of $r$ below which a $1 \sigma$-level detection becomes impossible even for maximal $P$ violation (when $\sigma_{\Delta \chi} \geq$ 1). This value is far above the current upper limit for WMAP-5 (compare to Ref. [13]), and so WMAP-5 can give no constraints on chiral gravity. Prospects are more optimistic for the next-generation CMB data releases. The critical $r$ is about 0.064 for SPIDER, 0.082 for Planck, 0.0079 for CMBPol, and 0.0023 for the $\mathrm{CV}$-limited experiment. If $r$ is just below the current detection limit of 0.22 [12], $\Delta \chi$ will be detectable at the $1 \sigma$ level if it is greater than $0.46,0.51,0.18$, and 0.11 for these four instruments, respectively. If we consider the $3 \sigma$ confidence level, the corresponding minimum detectable values are larger by a factor of $\sim 3$.

To conclude this section, we show how different multipoles $l$ contribute to the sum of Eq. (6), separating the contribution from $T B$ and $E B$, in Fig. 3. In this plot, only the $T B / E B$ summands of Eq. (6) are plotted against $l$, for $r=0.22$, for SPIDER, Planck, and CMBPol. The offdiagonal terms that contain the covariance between $T B$ and $E B$ are negligible. The major contribution to $\sigma_{\Delta \chi}^{-2}$ for all five experiments comes from the $T B$ power spectrum, from low multipoles, $l \sim 7$. Thus, large angular scales in

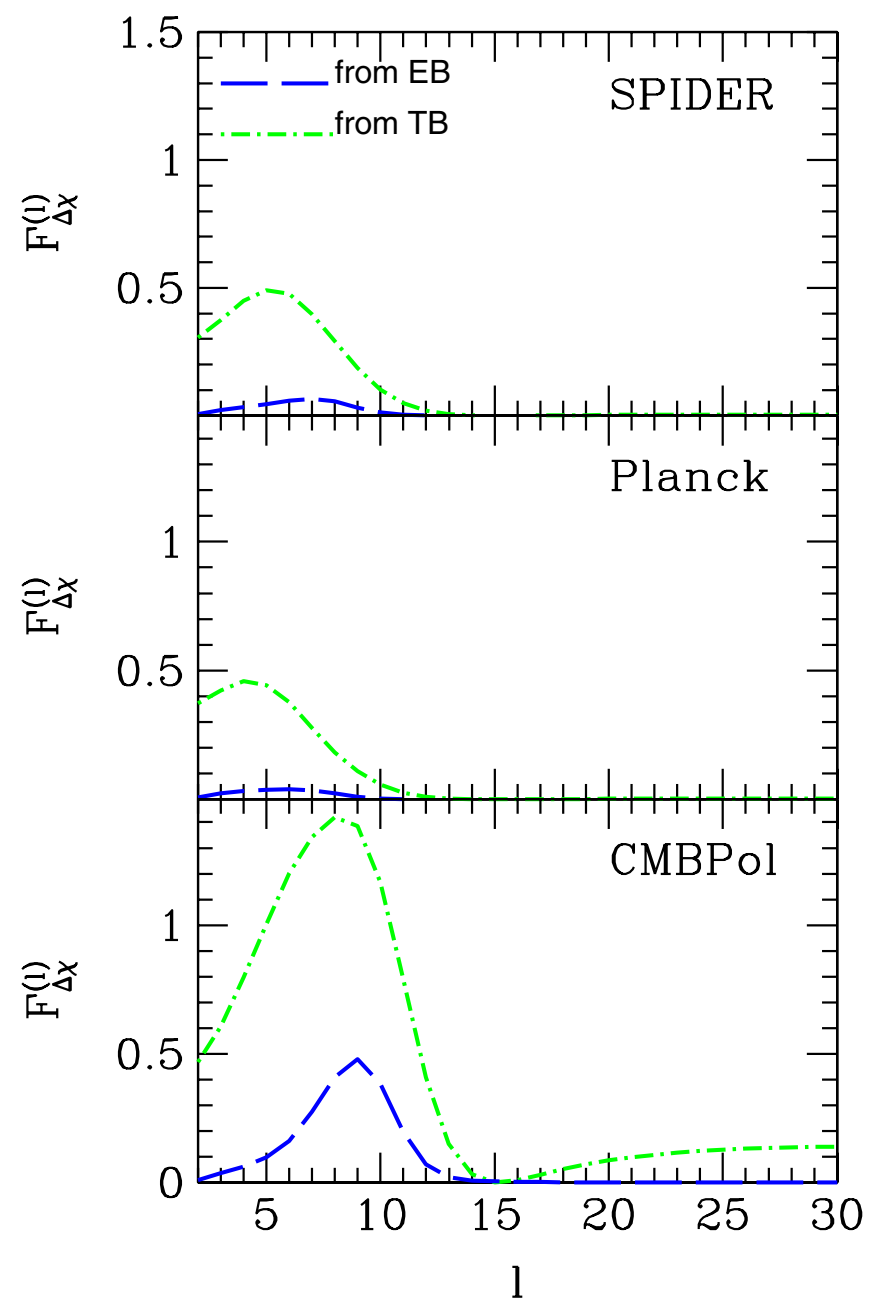

FIG. 3 (color online). Diagonal ( $T B, T B$ and $E B, E B)$ summands of Eq. (6), for $r=0.22$, are plotted against the multipole $l$ to show that the constraint to $\Delta \chi$ comes primarily from the $T B$ power spectrum at $l \sim 7$.

$T B$ (at $l \leq 10$ ) contain most of the information about gravitational chirality.

\section{CONSTRAINING COSMOLOGICAL BIREFRINGENCE}

Cosmological birefringence rotates the linear polarization at each point on the sky by an angle $\Delta \alpha$, and this rotation induces $T B / E B$ power spectra

$$
C_{l}^{T B, \text { rot }}=2 \Delta \alpha C_{l}^{T E}, \quad C_{l}^{E B, \text { rot }}=2 \Delta \alpha C_{l}^{E E} .
$$

One should note here that the additional $C_{l}^{E B \text {,rot }}$ due to rotation of a primordial $B$ mode into an $E$ mode is negligible, even if $r$ takes on its maximal value. Likewise, the additional $C_{l}^{E B}$ due to late-time rotation of a lensinginduced $B$ mode is also negligible (second order in small parameters). Since weak lensing (WL) does not induce $T B / E B$ correlations when averaged over the whole sky, 

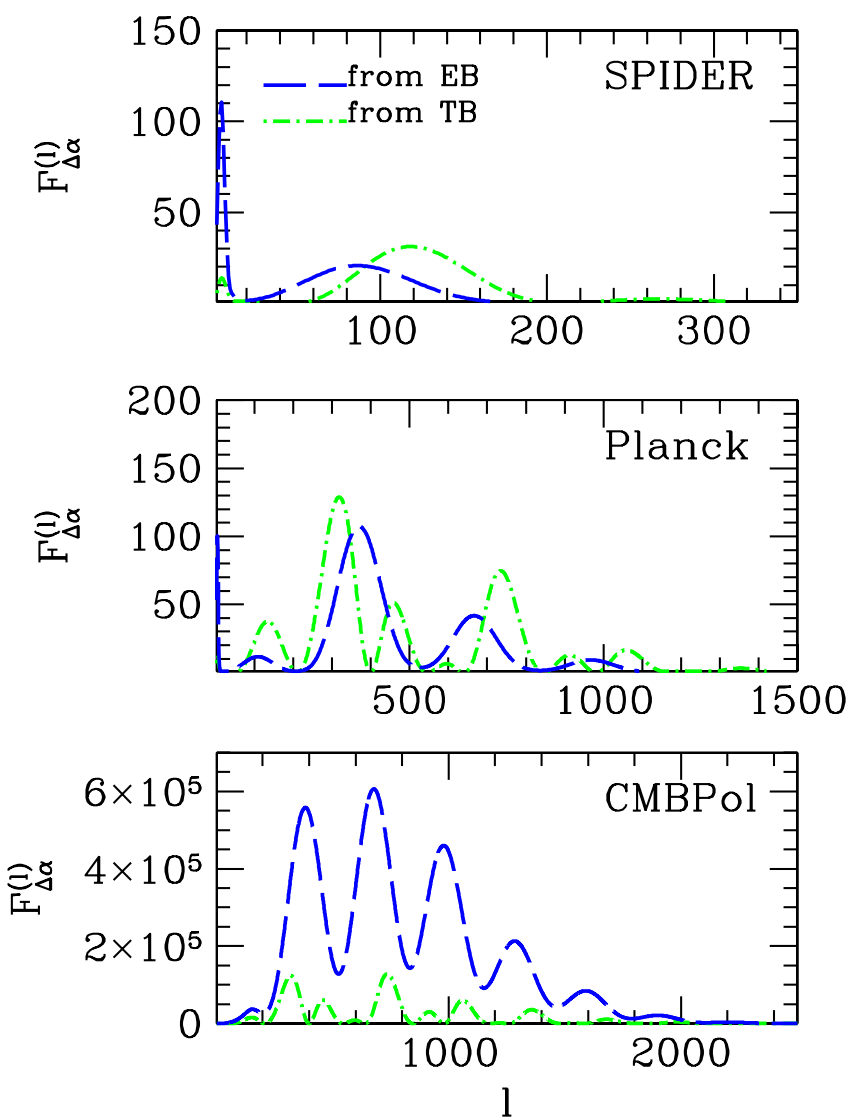

FIG. 4 (color online). Diagonal $(T B, T B$ and $E B, E B)$ summands of Eq. (9), for $r=0.22$, are plotted against the multipole $l$ to show that the constraints to $\Delta \alpha$ from future CMB experiments will come primarily from $l$ 's of $\sim 100,500$, or 700 (depending on the instrument).

this means that it does not contribute to the signal for CB, nor for the chiral GW. ${ }^{2}$

The error $\sigma_{\Delta \alpha}$ to which $\Delta \alpha$ can be measured is given by

$$
\sigma_{\Delta \alpha}^{-2}=\sum_{l} \sum_{A, A^{\prime}} \frac{\partial C_{l}^{A}}{\partial \Delta \alpha} \frac{\partial C_{l}^{A^{\prime}}}{\partial \Delta \alpha}\left[\Xi_{l}^{-1}\right]_{A A^{\prime}}
$$

Using the same instrumental parameters as in Sec. II B, and for $r=0.22$, we obtain the following $1 \sigma$ errors for the CB rotation angle: from WMAP-5, $3.2^{\circ}$; from SPIDER, $0.9^{\circ}$; from Planck, $15.9^{\prime}$; from CMBPol, $10.7^{\prime \prime}$; and from a $\mathrm{CV}$-limited experiment, $1.9 \mu \mathrm{arcs}$, in good agreement with previous forecasts [4,23-25].

In Fig. 4, we plot, separately, the contributions from only $T B$ and only $E B$ correlation to the sum in Eq. (9), as a function of multipole moment $l$, for the cases of SPIDER, Planck, and CMBPol, for $r=0.22$. The off-diagonal terms that contain the covariance between $T B$ and $E B$ are small. The dominant contribution to the constraint on $\Delta \alpha$ comes

\footnotetext{
${ }^{2} \mathrm{WL}$ does, however, contribute to the noise; numerically, this contribution turns out to be very small (see Sec. V).
}

from the $T B$ correlation for WMAP-5, and from $E B$ for the higher-precision instruments. Different multipoles give the leading summands in $\sigma_{\Delta \alpha}^{-2}$ for different instruments, but unlike the case of GW chirality, small angular scales $(l \gtrsim$ 100) always dominate the sum.

\section{SEPARATING GRAVITATIONAL CHIRALITY FROM COSMOLOGICAL BIREFRINGENCE}

In this section, we ask how well the effects of chiral gravity and $\mathrm{CB}$ can be distinguished, assuming that a $T B / E B$ correlation has been detected.

\section{A. First-order effects on the $E B$ and $T B$ correlations}

To first order in $\Delta \alpha$ and $\Delta \chi$, the $T B / E B$ power spectra are a sum of a part $C_{l}^{A \text {,chi }}$ due to chiral GWs and a part $C_{l}^{A \text {,rot }}$ due to $\mathrm{CB}$. The combined $E B$ and $T B$ power spectra can be written

$$
\begin{aligned}
& C_{l}^{T B, \mathrm{obs}}=\Delta \chi C_{l}^{T B, t}(\Delta \chi=1)+2 \Delta \alpha C_{l}^{T E}, \\
& C_{l}^{E B, \mathrm{obs}}=\Delta \chi C_{l}^{E B, t}(\Delta \chi=1)+2 \Delta \alpha C_{l}^{E E},
\end{aligned}
$$

where the superscript $t$ indicates the tensor-induced part of the power spectrum, while the absence of it denotes the full power spectrum, including the scalar part.

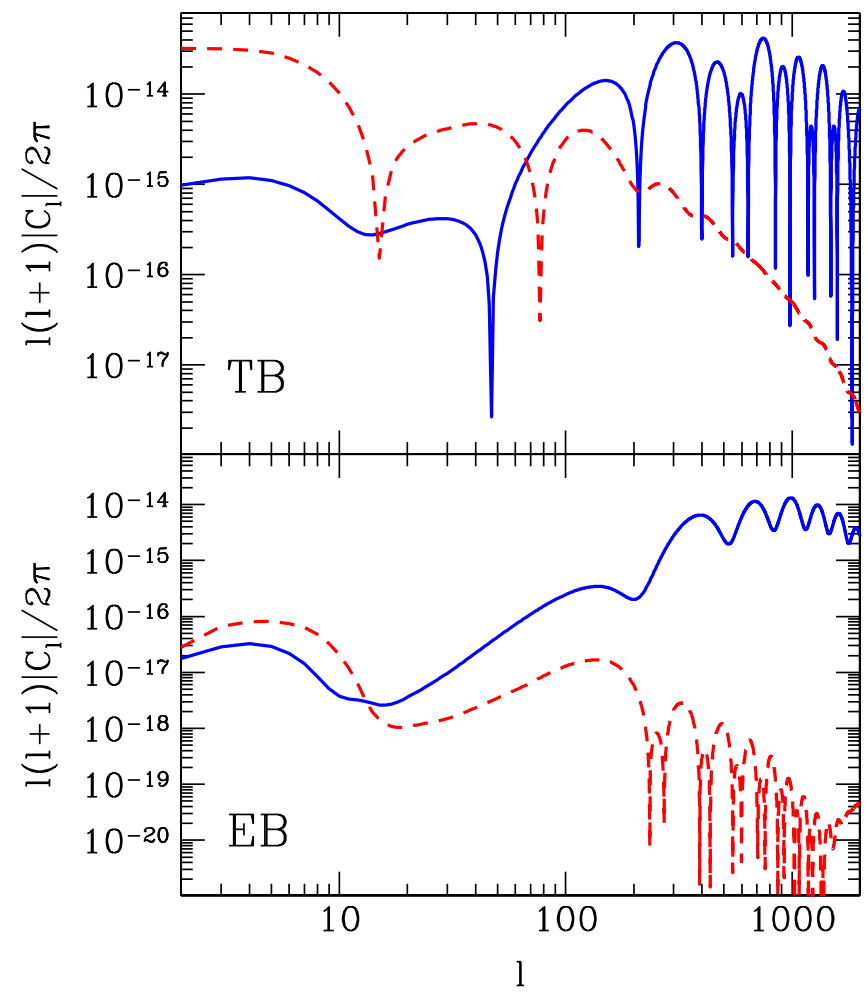

FIG. 5 (color online). We show $T B$ and $E B$ power spectra from chiral GWs for $\Delta \chi=0.2$ and $r=0.22$ (dashed red curves) and from cosmological birefringence for $\Delta \alpha=5^{\prime}$ (solid blue curves). 
Figure 5, which shows $C_{l}^{A, \text { chi }}$ and $C_{l}^{A, \text { rot }}$, demonstrates that the contributions from these two mechanisms are qualitatively different. Our goal now is to quantify how well they can be distinguished, given the finite precision of the temperature/polarization maps.

The Fisher matrix for $\Delta \alpha$ and $\Delta \chi$ has the following entries (see [7]):

$$
\mathcal{F}_{i j}=\sum_{l} \sum_{A, A^{\prime}} \frac{\partial C_{l}^{A}}{\partial a_{i}} \frac{\partial C_{l}^{A^{\prime}}}{\partial a_{j}}\left[\Xi_{l}^{-1}\right]_{A A^{\prime}}
$$

where $i, j=\{1,2\} ; a_{i}$ and $a_{j}$ are the elements of $\vec{a}=$ $(\Delta \alpha, \Delta \chi) ; A, A^{\prime}=\{T B, E B\}$; and $\mathcal{F}$ is the inverse of the covariance matrix between $\Delta \alpha$ and $\Delta \chi$. The derivatives in Eq. (11) can be calculated using Eq. (10), and $\left[\Xi^{-1}\right]_{A A^{\prime}}$ is the inverse of the $T B-E B$ covariance matrix given by Eq. (A5) of Appendix A. Once again, we employ the null hypothesis $^{3} \Delta \alpha=\Delta \chi=0$.

\section{B. Numerical results: Constraints on the $\Delta \alpha-\Delta \chi$ parameter space}

Figure 6 shows $1 \sigma$ error ellipses in the $\Delta \alpha-\Delta \chi$ parameter space, for the null hypothesis $\Delta \alpha=\Delta \chi=0$, with WMAP-5, SPIDER, Planck, CMBPol, and a CV-limited experiment, for a range of tensor-to-scalar ratios. In addition, each plot shows a $1 \sigma$-error ellipse for a different set of fiducial values: $\Delta \chi=0.2$ and $\Delta \alpha=5^{\prime \prime}$. The ellipses for this model are merely shifted in the $\Delta \alpha-\Delta \chi$ space, but are otherwise not significantly different from the nullhypothesis ellipses. From Fig. 6, we see that once we take into account the covariance between $\Delta \alpha$ and $\Delta \chi$, the results differ very slightly from the two cases where we had only one of the $P$-violating mechanisms acting on the CMB. This is clear from the fact that the ellipses show very little tilt in $\Delta \alpha-\Delta \chi$ space. We conclude that if nonvanishing $T B / E B$ correlations are detected with high statistical significance, we will be able to distinguish CB from gravitational chirality to a high degree.

This result can also be explained from Figs. 3 and 4, which show that the $\Delta \chi$ constraint comes primarily from $T B$ at low l's, while the $\Delta \alpha$ constraint comes primarily from $E B$ at high $l$ 's.

\section{SUMMARY AND CONCLUSIONS}

In this paper, we first revisit the sensitivity of current and future $\mathrm{CMB}$ experiments to gravitational chirality and to

\footnotetext{
${ }^{3}$ Even in the case where we work around nonzero fiducial values, the effect of the off-diagonal terms is negligible and the covariance matrix can be treated as a block-diagonal matrix to good precision. In addition, the cross terms between $T B / E B$ and the other four power spectra in Eq. (11) vanish, to the first order in small parameters, so we really only need to consider $T B$ and $E B$.
}

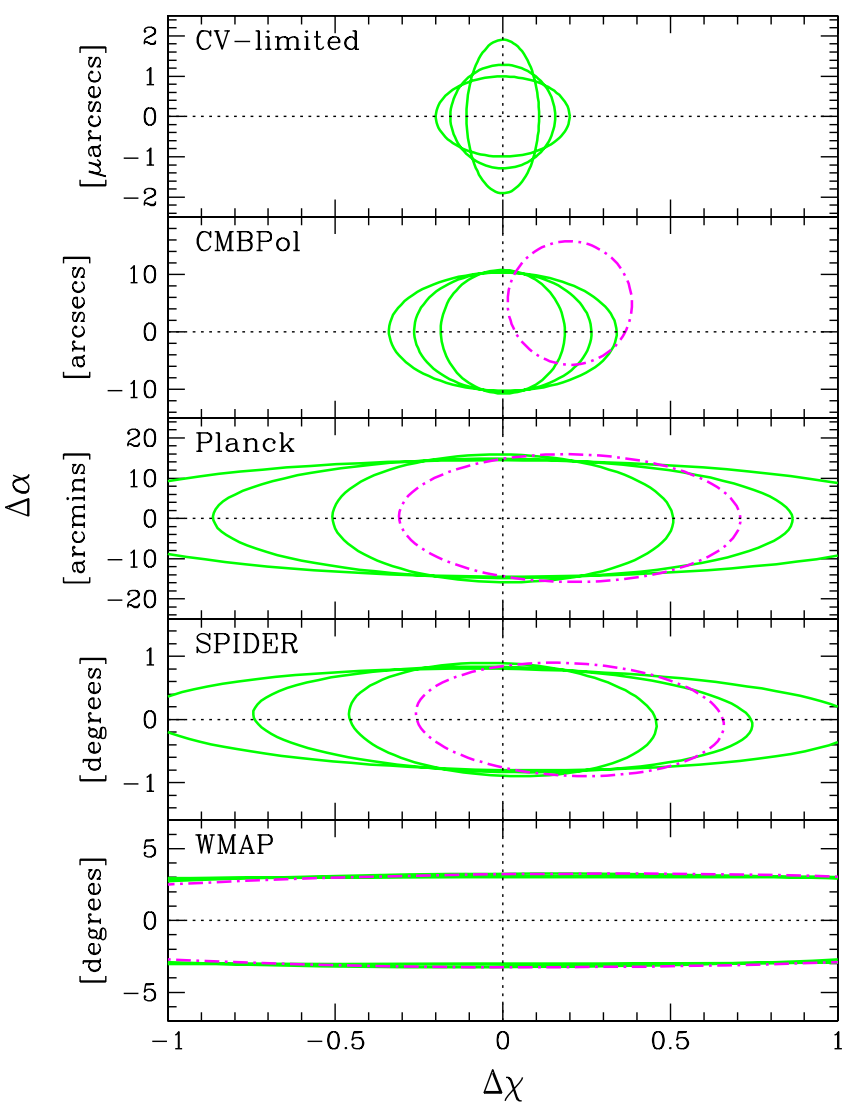

FIG. 6 (color online). Constraints on the allowed $\Delta \alpha-\Delta \chi$ parameter space are shown for the case of null detection with different instruments. The solid-line ellipses on each plot are for the fiducial value of zero for both parameters, and for the following values of tensor-to-scalar ratios (going from the narrowest to the widest ellipse in the $\Delta \chi$ direction): $0.22,0.1$, and 0.06. The dot-dashed ellipse in each plot is for $r=0.22$, for a model with $\Delta \chi=0.2$ and $\Delta \alpha=5^{\prime \prime}$. In both models, the tilt of the ellipses is almost negligible, which means that the two $P$-violating mechanisms are separable to high accuracy, provided there is detection with high statistical significance. Thus, the constraints on both parameters are almost the same as those calculated in the previous sections.

cosmological birefringence, separately. We show that the WMAP-5 polarization data are not precise enough to provide any information about gravitational chirality, even for the case where the tensor-to-scalar ratio is just below the current upper constraint of 0.22. Planck and SPIDER may be able to make a marginal detection, but only if $r$ and $\Delta \chi$ are both close to their maximal allowed values. CMBPol may probe gravitational chirality over a larger range of the $r-\Delta \chi$ parameter space. As an illustration, the smallest amount of GW chirality detectable at the $3 \sigma$ level with a cosmic-variance-limited experiment, if $r$ is just below 0.22 , corresponds to about $65 \%$ of the GW background being of one handedness, and 35\% of another. In an analogous analysis, we show that Planck has a $1 \sigma$ sensitivity to a 
CB rotation angle of about $16^{\prime}$, while a CV-limited experiment could reach about $2 \mu$ arcs.

In the second part of this paper we show that there is no strong degeneracy between $\Delta \alpha$ and $\Delta \chi$ parameters. In other words, the effects of chiral gravity and $\mathrm{CB}$ can be easily distinguished, provided that the $T B / E B$ correlations are clearly detected. However, the same results can be interpreted as to infer that a marginal (e.g., $3 \sigma$ ) detection of $\Delta \alpha$ could be due, alternatively, to gravitational chirality at some level. For example, if CMBPol were to measure $\Delta \alpha \simeq 15^{\prime \prime}$ and find $r=0.1$, that $T B / E B$ detection could alternatively be attributed, with similar statistical significance, to gravitational chirality with $\Delta \chi=0.6$. If, however, the earlier suggestion of a $T B / E B$ signal corresponding to a rotation angle of $6^{\circ}$ had held up, it could not have been attributed to chiral GWs, as the implied value of $\Delta \chi$ would have been in the unphysical regime $\Delta \chi \gg 1$.

If a parity-violating signal is detected in the CMB and attributed to $\mathrm{CB}$, it may be possible to test it further with observations of cosmological radio sources [26]. Offdiagonal correlations in the CMB may also provide additional information on $\mathrm{CB}$, if the $\mathrm{CB}$ rotation angle is position dependent [23-25], as suggested in Ref. [27]. A parity-violating signal from chiral GWs might be distinguished from that due to $\mathrm{CB}$ through direct detection of the gravitational-wave background at shorter wavelengths [28]. Finally, it may be that any signals of chiral gravity in the CMB may be corroborated, within the context of specific alternative-gravity theories, by a variety of other observations and measurements [29].

Finally, let us emphasize that we do not expect weak lensing by the large-scale structure ${ }^{4}$ to be a significant contaminant in detecting a signal from chiral gravity and/ or cosmological birefringence, with Planck or CMBPol. In the case of chiral GW, most of the information is carried by the large-scale inhomogeneities, where WL has no power. On the other hand, for $\mathrm{CB}$, the corresponding WL correction to the variance turns out to be numerically small: it is less than $10 \%$ for CMBPol and negligible for all other instruments we considered (except for a CV-limited experiment where we analyzed unlensed maps only); for more details, see Appendix A.

\section{ACKNOWLEDGMENTS}

V. G. thanks Timothy Morton for useful comments. This work was supported by DoE DE-FG03-92-ER40701 and the Gordon and Betty Moore Foundation.

\footnotetext{
${ }^{4} \mathrm{WL}$ is another source of $B$ modes in $\mathrm{CMB}$ polarization maps, but it does not induce $T B / E B$ correlations. Also, the WLinduced $B$ modes are of different nature than those sourced by the cosmological birefringence and chiral gravity; for details, see Appendix B of Ref. [25].
}

\section{APPENDIX A: POWER-SPECTRA COVARIANCE MATRIX}

Suppose we have obtained multipole coefficients $d_{l m}^{X^{\prime}}$, for $X=\{T, E, B\}$ from a full-sky CMB map. Their variance is given by [7]

$$
\left\langle\left(d_{l m}^{X}\right)^{*} d_{l^{\prime} m^{\prime}}^{X^{\prime}}\right\rangle=\left(\left|W_{l}^{b}\right|^{2} C_{l}^{X X^{\prime}}+w_{X X^{\prime}}^{-1}\right) \delta_{l l^{\prime}} \delta_{m m^{\prime}},
$$

where $C_{l}^{X X^{\prime}}$ is the power spectrum of the signal, $W_{l}^{b} \simeq$ $\exp \left(-l^{2} \sigma_{b}^{2} / 2\right)$ is the window function to take into account the effects of beam smearing, and $\sigma_{b} \equiv \theta_{\mathrm{FWHM}} / \sqrt{8 \ln (2)}$ with $\theta_{\text {FWHM }}$ the beam width. The $w_{X X}^{-1}$ are the contributions to the measured power spectra due to instrumental noise; they are given by

$$
w_{T T}^{-1} \equiv \frac{4 \pi \sigma_{T}^{2}}{N_{\mathrm{pix}}}, \quad \text { and } \quad w_{E E}^{-1}=w_{B B}^{-1} \equiv \frac{4 \pi \sigma_{P}^{2}}{N_{\mathrm{pix}}} .
$$

Here, $\sigma_{T}$ and $\sigma_{P}$ are the pixel noise in temperature and polarization, respectively, and $N_{\text {pix }}=4 \pi \theta_{\mathrm{FWHM}}^{-2}$ is the number of pixels. We assume that the signal is not correlated to the noise, and that the noise in the polarization is not correlated to the noise in the temperature; i.e. $w_{E T}^{-1}=$ $w_{B T}^{-1}=w_{E B}^{-1}=0$.

The estimators for the power spectrum are then

$$
\hat{C}_{l}^{X X^{\prime}}=\left|W_{l}^{b}\right|^{-2}\left(\sum_{m=-l}^{l} \frac{\left(d_{l m}^{X}\right)^{*} d_{l m}^{X^{\prime}}}{(2 l+1)}-w_{X X^{\prime}}^{-1}\right),
$$

and the power-spectrum covariance matrix is then given by

$$
\begin{aligned}
\Xi_{l}^{X_{1} X_{2}, X_{3} X_{4}} \equiv & \left\langle\left(\hat{C}_{l}^{X_{1} X_{2}}-C_{l}^{X_{1} X_{2}}\right)\left(\hat{C}_{l}^{X_{3} X_{4}}-C_{l}^{X_{3} X_{4}}\right)\right\rangle \\
= & \left\langle\hat{C}_{l}^{X_{1} X_{2}} \hat{C}_{l}^{X_{3} X_{4}}\right\rangle-C_{l}^{X_{1} X_{2}} C_{l}^{X_{3} X_{4}} \\
= & \left|W_{l}^{b}\right|^{-4}\left(\sum_{m, m^{\prime}} \frac{\left\langle\left(d_{l m}^{X_{1}}\right)^{*} d_{l m}^{X_{2}}\left(d_{l m^{\prime}}^{X_{3}}\right)^{*} d_{l m^{\prime}}^{X_{4}}\right\rangle}{(2 l+1)^{2}}\right. \\
& \left.-\left\langle\left(d_{l m}^{X_{1}}\right)^{*} d_{l^{\prime} m^{\prime}}^{X_{2}}\right\rangle\left\langle\left(d_{l m}^{X_{3}}\right)^{*} d_{l^{\prime} m^{\prime}}^{X_{4}}\right)\right) \\
= & \frac{\left|W_{l}^{b}\right|^{-4}}{(2 l+1)}\left(\left\langle\left(d_{l m}^{X_{1}}\right)^{*} d_{l^{\prime} m^{\prime}}^{X_{3}}\right\rangle\left\langle\left(d_{l m}^{X_{2}}\right)^{*} d_{l^{\prime} m^{\prime}}^{X_{4}}\right\rangle\right. \\
& \left.+\left\langle\left(d_{l m}^{X_{1}}\right)^{*} d_{l^{\prime} m^{\prime}}^{X_{4}}\right\rangle\left\langle\left(d_{l m}^{X_{2}}\right)^{*} d_{l^{\prime} m^{\prime}}^{X_{3}}\right)\right) .
\end{aligned}
$$

Using Eqs. (A1) and (A4) we get

$$
\Xi_{l}^{X_{1} X_{2}, X_{3} X_{4}}=\frac{1}{(2 l+1)}\left(\tilde{C}_{l}^{X_{1} X_{3}} \tilde{C}_{l}^{X_{2} X_{4}}+\tilde{C}_{l}^{X_{1} X_{4}} \tilde{C}_{l}^{X_{2} X_{3}}\right),
$$

where

$$
\tilde{C}_{l}^{X X^{\prime}} \equiv C_{l}^{X X^{\prime}}+w_{X X^{\prime}}^{-1}\left|W_{l}^{b}\right|^{-2} .
$$

For $X X^{\prime}=B B$, both the primordial contribution and the contribution from the $B$ modes induced by the $\mathrm{WL}^{5}$ are

\footnotetext{
${ }^{5}$ The lensing surface is assumed to be located after the LSS, and before most of the rotation by $\mathrm{CB}$ can take place.
} 


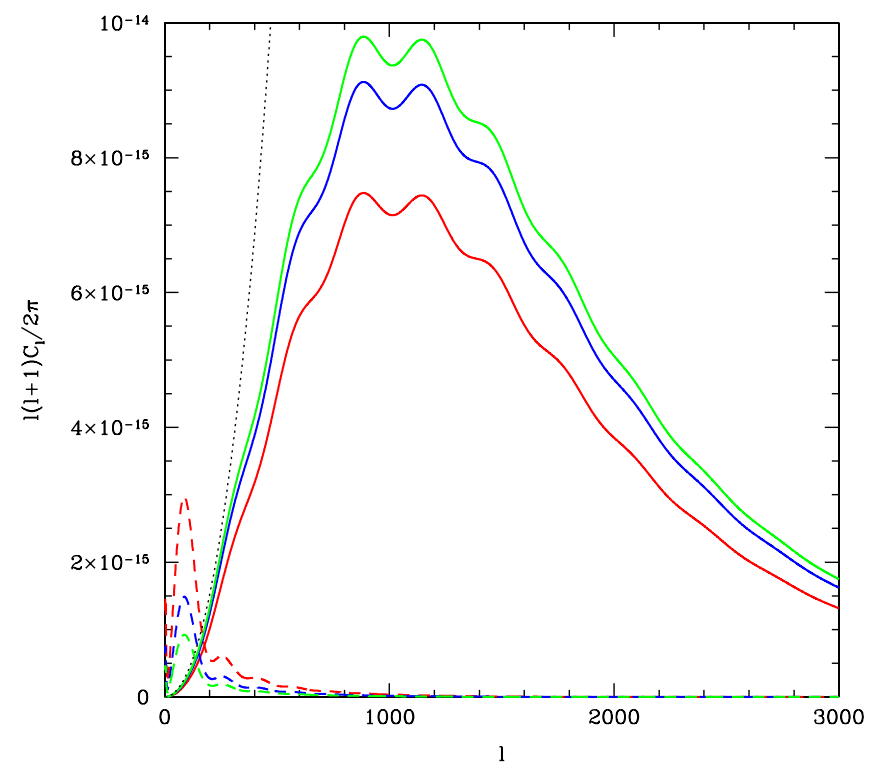

FIG. 7 (color online). $\quad B B$ power spectra from primordial GWs (dashed lines), and from WL of the primordial $E$ modes (solid lines), for $r=0.22$ (red, bottom solid and top dashed lines), $r=$ 0.1 (blue, middle lines), and $r=0.06$ (green, top solid and bottom dashed lines). The dotted black line is the appropriately normalized noise power spectrum for CMBPol. Note that the noise dominates above $l \sim 100$.

taken into account on the right-hand side of the above equation, for the case of $\mathrm{CB}$. This is done for all the experiments, with the exception of a CV-limited experiment, where we investigate the limiting case of fully unlensend maps. For the case of chiral GW, there is no need to include WL, since the constraints to $\Delta \chi$ come from large scales (below $l$ of 10 or so), where WL has no power. To illustrate how different terms of Eq. (A5) contribute to the overall sum, we show the primordial and lensing-induced $B B$ power spectra for a range of $r$ 's, and the noise power spectrum for CMBPol (normalized accordingly), in Fig. 7. The lensed maps are obtained from the CMBFAST code, for the same set of cosmological parameters we use throughout this paper.

To account for partial-sky coverage, we add a factor $f_{\text {sky }}^{-1}$ (the inverse of the fraction of the sky used in the analysis) to the right-hand side of Eq. (A5). We also multiply the factors $w_{X X}^{-1}$ in Eq. (A6) by a factor $\left(f_{\text {sky }}^{0}\right)^{-1}$ (the inverse of the fraction of the sky surveyed).

\section{APPENDIX B: CONSTRAINTS ON TENSOR-TO- SCALAR RATIO}

Here we examine a claim of Ref. [5] that if the GW background is chiral, it may be more easily detected through the $T B$ signal than the $B B$ signal, the reason being that it may be easier to detect a weak signal $(B)$ by cross correlation with a strong one $(T)$ than against itself.
Under the null hypothesis (no GWs), the error with which the tensor-to-scalar ratio can be measured, from just one power spectrum $A$ (where $A$ is $B B, T B$, or $E B$ ) is

$$
\sigma_{r}^{-2}=\sum_{l}\left(\frac{\partial C_{l}^{A}}{\partial A_{t}}\right)^{2}\left(\Xi_{l, A A}\right)^{-1}
$$

Given that the power spectra are simply proportional to $A_{t}$, $\left(\partial C_{l}^{A} / \partial A_{t}\right) \propto C_{l}^{A}$. The relevant covariance-matrix entries are

$$
\begin{gathered}
\Xi_{l}^{B B}=\frac{2}{2 l+1}\left(\tilde{C}_{l}^{B B}\right)^{2}=\frac{2}{2 l+1} w_{B B}^{-1}\left(W_{l}^{b}\right)^{-2}, \\
\Xi_{l}^{T B}=\frac{1}{2 l+1}\left[\left(\tilde{C}_{l}^{T B}\right)^{2}+\tilde{C}_{l}^{T T} \tilde{C}_{l}^{B B}\right] \\
=\frac{1}{2 l+1} w_{B B}^{-1}\left(W_{l}^{b}\right)^{-2}\left[C_{l}^{T T, s}+w_{T T}^{-1}\left(W_{l}^{b}\right)^{-2}\right],
\end{gathered}
$$

(and similarly for $E B$, with $T \rightarrow E$ ) where we have employed the null hypothesis in the second equality in each of these equations.

Given that $w_{T T}^{-1} \ll C_{l}^{T T}$ already from current data for the low $l$ at which the GW signal arises, we can set $w_{T T}^{-1}=0$. Moreover, $C_{l}^{T B} \sim \beta\left(C_{l}^{B B} C_{l}^{T B}\right)^{1 / 2}$, with $\beta \sim 0.1$. As a result, while the summand for $\sigma_{r}^{-2}$ from $B B$ is $\sim\left(C_{l}^{B B}\right)^{2} / w_{B B}^{-2}$, that from $T B$ is $\sim C_{l}^{B B} / w_{B B}^{-1}$. Thus, in the limit of sufficiently high signal-to-noise, $w_{B B}^{-1} \rightarrow 0$, the $B B$ signal provides a better probe (smaller $\sigma_{r}$ ). In other words, the value of the cross correlation with $T$ is ultimately limited by cosmic variance (as is also the cross correlation with $E$ ), while the $B B$ sensitivity improves without limit as the instrumental noise is reduced. (The importance of $T B$ is also weakened slightly given that $C_{l}^{T B}<\left[C_{l}^{T T} C_{l}^{B B}\right]^{1 / 2}$.) It is true that in the opposite limit, where $w_{B B}^{-1}$ is large, $T B$ is more sensitive to GWs (with $\Delta \chi=1$ ) than $T T$. However, this limit is only of academic interest, as it encompasses the regime of $r$ that is already ruled out by temperature measurements.

To make these arguments more quantitatively precise, we have evaluated $\sigma_{r}$ for $B B, T B$, and $E B$ (for $\Delta \chi=1$ ) for WMAP-5, SPIDER, Planck, and CMBPol; the results are shown in Table II. We see that the sensitivity to GWs with future experiments will come primarily from $B B$, with only

TABLE II. The error $\sigma_{r}$ on the tensor-to-scalar ratio for a chiral GW background with $\Delta \chi=1$ from $B B, T B$, and $E B$ for several $\mathrm{CMB}$ experiments.

\begin{tabular}{lccc}
\hline \hline Instrument & From $B B$ & From $T B$ & From $E B$ \\
\hline WMAP-5 & 0.68 & 0.37 & 3.03 \\
SPIDER & 0.011 & 0.051 & 0.20 \\
Planck & 0.026 & 0.071 & 0.30 \\
CMBPol & $1.57 \times 10^{-5}$ & 0.0018 & 0.0062 \\
\hline \hline
\end{tabular}


marginal improvement from $T B$. While the $T B$ sensitivity of WMAP-5 is better than that from $B B$, the smallest $r$ detectable with either is already larger than the upper limit from $T T$.
The bottom line: While $T B$ may improve the sensitivity to a chiral-GW background, it does so only marginally, with most of the sensitivity due primarily to $B B$ (see also Ref. [13]).
[1] A. H. Guth, Phys. Rev. D 23, 347 (1981); A. Albrecht and P. J. Steinhardt, Phys. Rev. Lett. 48, 1220 (1982); A. D. Linde, Phys. Lett. 108B, 389 (1982).

[2] A. G. Riess et al. (Supernova Search Team Collaboration), Astron. J. 116, 1009 (1998).

[3] S. M. Carroll, Phys. Rev. Lett. 81, 3067 (1998).

[4] A. Lue, L. M. Wang, and M. Kamionkowski, Phys. Rev. Lett. 83, 1506 (1999).

[5] C. R. Contaldi, J. Magueijo, and L. Smolin, Phys. Rev. Lett. 101, 141101 (2008).

[6] T. Takahashi and J. Soda, Phys. Rev. Lett. 102, 231301 (2009).

[7] M. Kamionkowski, A. Kosowsky, and A. Stebbins, Phys. Rev. D 55, 7368 (1997); Phys. Rev. Lett. 78, 2058 (1997).

[8] M. Zaldarriaga and U. Seljak, Phys. Rev. D 55, 1830 (1997); U. Seljak and M. Zaldarriaga, Phys. Rev. Lett. 78, 2054 (1997).

[9] N. F. Lepora, arXiv:gr-qc/9812077.

[10] B. Feng et al., Phys. Rev. Lett. 96, 221302 (2006).

[11] T. Kahniashvili, R. Durrer, and Y. Maravin, Phys. Rev. D 78, 123009 (2008); J. Q. Xia et al., Astrophys. J. 679, L61 (2008); Astron. Astrophys. 483, 715 (2008); E. Y. Wu et al. (QUaD Collaboration), Phys. Rev. Lett. 102, 161302 (2009); L. Pagano et al., Phys. Rev. D 80, 043522 (2009).

[12] E. Komatsu et al. (WMAP Collaboration), Astrophys. J. Suppl. Ser. 180, 330 (2009).

[13] S. Saito, K. Ichiki, and A. Taruya, J. Cosmol. Astropart. Phys. 09 (2007) 002.

[14] http://map.gsfc.nasa.gov/.
[15] B. P. Crill et al., arXiv:0807.1548.

[16] http://planck.caltech.edu/.

[17] http://cmbpol.uchicago.edu/.

[18] G. Jungman, M. Kamionkowski, A. Kosowsky, and D. N. Spergel, Phys. Rev. D 54, 1332 (1996); Phys. Rev. Lett. 76, 1007 (1996).

[19] U. Seljak and M. Zaldarriaga, Astrophys. J. 469, 437 (1996).

[20] A. R. Pullen and M. Kamionkowski, Phys. Rev. D 76, 103529 (2007).

[21] http://www.rssd.esa.int/PLANCK.

[22] J. Bock et al., arXiv:0805.4207.

[23] M. Kamionkowski, Phys. Rev. Lett. 102, 111302 (2009).

[24] A.P. S. Yadav, R. Biswas, M. Su, and M. Zaldarriaga, Phys. Rev. D 79, 123009 (2009).

[25] V. Gluscevic, M. Kamionkowski, and A. Cooray, Phys. Rev. D 80, 023510 (2009).

[26] J. F. C. Wardle, R. A. Perley, and M. H. Cohen, Phys. Rev. Lett. 79, 1801 (1997); J.P. Leahy, arXiv:astro-ph/ 9704285; B. Nodland and J. P. Ralston, Phys. Rev. Lett. 78, 3043 (1997); D. J. Eisenstein and E. F. Bunn, Phys. Rev. Lett. 79, 1957 (1997); S. M. Carroll and G. B. Field, Phys. Rev. Lett. 79, 2394 (1997).

[27] M. Pospelov, A. Ritz, and C. Skordis, Phys. Rev. Lett. 103, 051302 (2009); M. Li and X. Zhang, Phys. Rev. D 78, 103516 (2008).

[28] N. Seto and A. Taruya, Phys. Rev. D 77, 103001 (2008); Phys. Rev. Lett. 99, 121101 (2007); N. Seto, Phys. Rev. D 75, 061302 (2007); Phys. Rev. Lett. 97, 151101 (2006).

[29] S. Alexander and N. Yunes, Phys. Rep. 480, 1 (2009). 